\title{
Produits nouveaux
}

\section{Le système DOSICARD}

\author{
F. LACOSTE, M. LUCAS*
}

(Manuscrit reçu le 7 décembre 1992)

RÉSUMÉ DOSICARD est un dosimètre électronique de rayonnements $X$ et gamma de la dimension d'une carte de crédit. II est destiné à être porté par les travailleurs des installations nucléaires civiles ou militaires, des centres de recherche et des milieux médicaux. DOSICARD permet une gestion souple et le suivi en temps réel des expositions aux rayonnements de ces personnes. Des alarmes sonore et visuelle correspondant à des seuils de dose ou de débit de dose en font un appareil de protection efficace sur les lieux de travail. DOSICARD peut être utilisé de manière autonome ou en relation avec un système informatique qui permet un traitement collectif des informations.

ABSTRACT DOSICARD is an $\mathrm{X}$ and gamma radiation solid state dosemeter available in a credit card format. It is to be worn by nuclear civilian or defence personnel, in research labs or in the medical field. DOSICARD allows flexible management and real time monitoring of its bearer's radiation exposure. Audible and visual warnings corresponding to preset exposure levels or dose rates make DOSICARD a dependable protection device in the work environment. It can be used as a stand-alone equipment or in association with a data processing system in case of large institutions.

\section{Introduction}

Le système DOSICARD est un système de dosimétrie $X, \gamma$ et $B$ de haute énergie qui repose sur l'utilisation de badges électroniques personnels, associés à un environnement informatique pour une gestion collective des données (fig. 1 et 2). Il est destiné aux travailleurs des installations nucléaires civiles ou militaires, des centres de recherche et des milieux médicaux. Le badge DOSICARD mesure, en temps réel, les équivalents de dose reçus et déclenche des alarmes sonores et visuelles en cas de dépassement de seuils autorisés, conditions nécessaires à une prévention efficace. De plus, DOSICARD cumule et mémorise ces équivalents de dose sur des périodes de référence (mois, trimestre, an, 5 ans) réalisant ainsi, de façon autonome, une gestion de I'historique des doses.

* Nomatek, 12 avenue des Prés, 78180 Montigny-le-Bretonneux. 


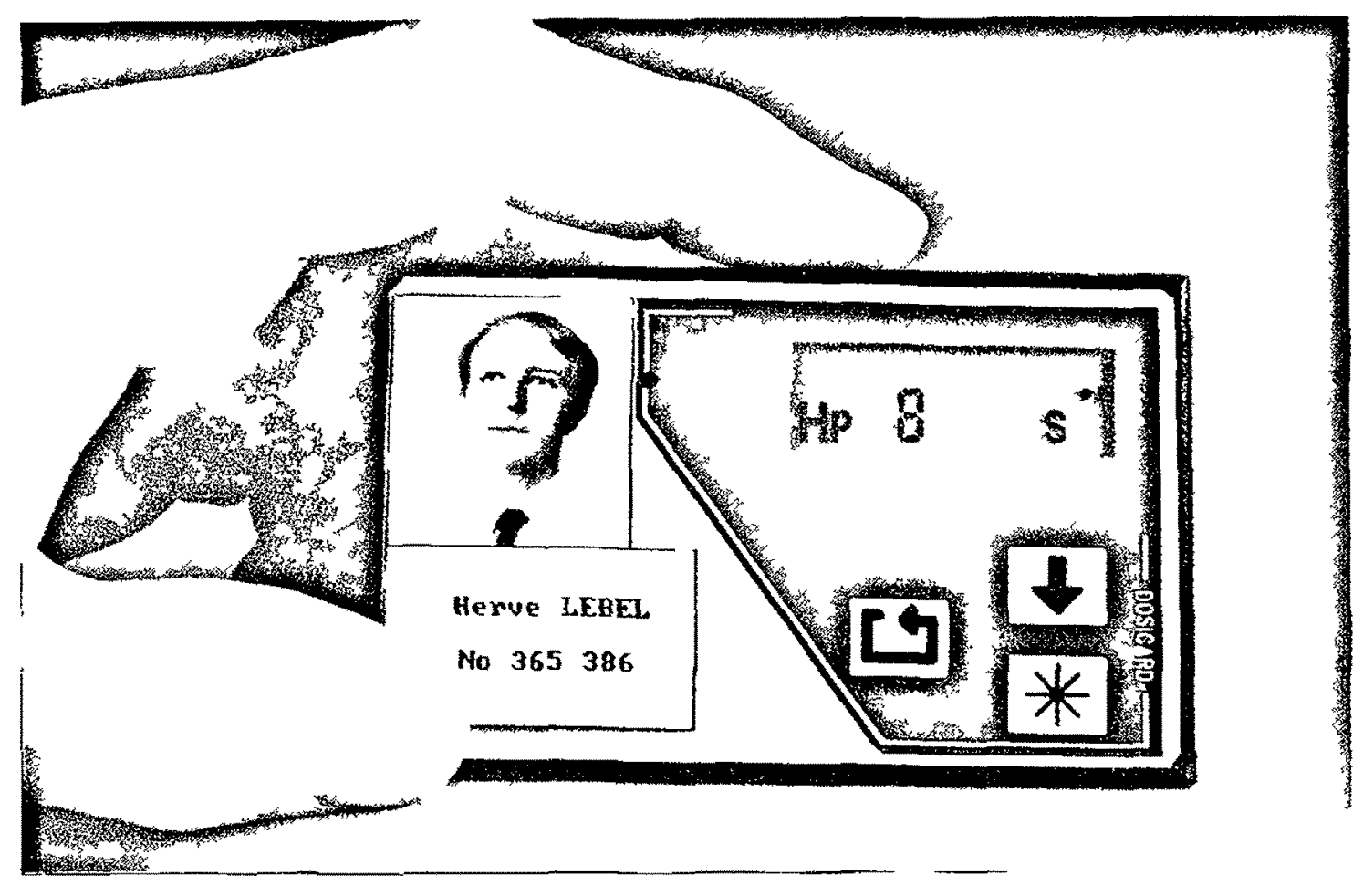

Fig. 1. - Dosimètre - badge DOSICARD.

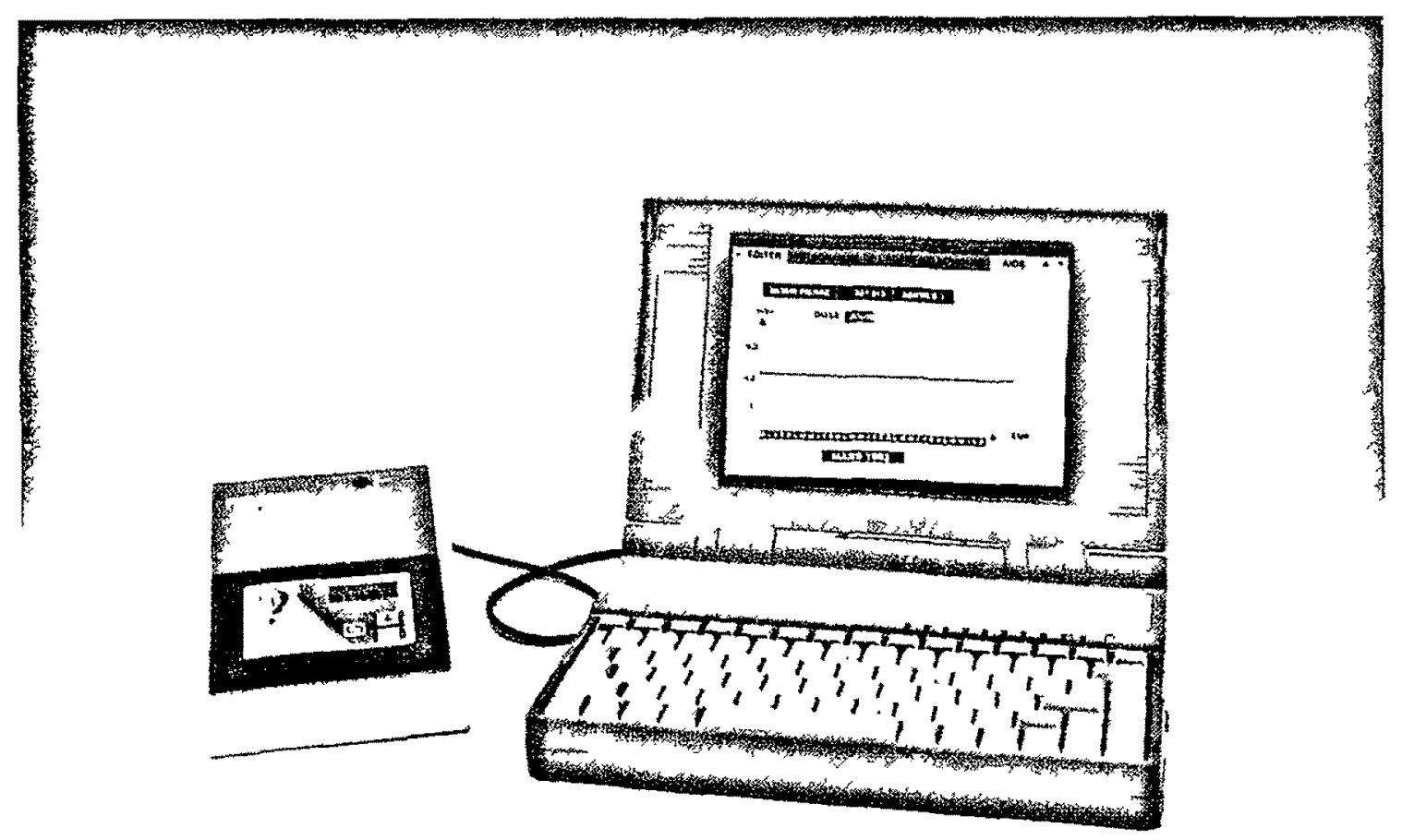

Fig. 2. - Sous-système d'attribution de badges (SAB) : version portable. 
La disponibilité dans un badge de ces informations individuelles permet, en particulier, le suivi dosimétrique des personnels itinérants intervenant, par exemple, dans un pays étranger ou travaillant dans des sites géographiquement distincts ; DOSICARD apporte une réponse technique à cette question qui constitue, en particulier, une préoccupation actuelle des instances européennes. Par ailleurs, la connaissance directe, par le porteur, du cumul des doses qu'il a reçues est certainement un élément important dans la réduction des doses reçues par la sensibilisation du travailleur à son niveau d'exposition aux rayonnements.

\section{Description du système}

\subsection{Badge DOSICARD}

Le badge est d'un format type carte de crédit et de quelques millimètres d'épaisseur ; il est contenu dans une pochette transparente qui possède un système d'agrafage et permet de faciliter une décontamination éventuelle.

L'architecture électronique du badge, organisée autour d'un microcontrôleur, lui confère les fonctions suivantes (tab. I) :

a) Mesure d'équivalent de dose et de débit d'équivalent de dose : le badge mesure les rayonnements en terme d'équivalent de dose profonde Hp (10), grandeur opérationnelle définie par l'ICRU ; celle-ci correspond à l'équivalent de dose dans le tissu à la profondeur de $10 \mathrm{~mm}$, appropriée aux rayonnements fortement pénétrants. Le capteur utilisé est de type état solide constitué pratiquement d'une diode silicium équipée d'un dispositif de compensation en énergie.

b) Alarmes sonore et visuelle: les alarmes sont de 2 types:

- sonore par activation d'un "buzzer" dont le motif temporel est fonction de l'alarme (dose ou débit de dose) ;

- visuel par clignotement d'une diode électroluminescente située en partie supérieure du badge.

Les alarmes sont programmables et peuvent être positionnées soit par rapport à des critères opérationnels, soit par rapport à des seuils normalisés.

c) Mémorisation des informations : les informations sont stockées dans une mémoire non volatile (EEPROM) qui conserve celles-ci même en l'absence d'alimentation (ex. : pile déchargée), sur une durée de l'ordre de 10 ans. Les informations sont disponibles dès la remise sous tension du dispositif. Les informations peuvent être classées de la façon suivante :

- données d'identification du porteur telles que : nom, prénom, numéro d'identification, date et lieu de naissance, sexe, nationalité, nom de l'entreprise, catégorie, numéro SCPRI ; 
- données de dosimétrie telles que : code de travail, date, lieu d'intervention, doses cumulées (jour, mois, 60 derniers jours, trimestre, 12 derniers mois, an, 5 ans, carrière) ;

- données d'identification du badge telles que : numéro de série, date d'étalonnage, date de fin de validité, date de changement de pile.

d) Communication : le badge permet deux modes de communications:

- avec le porteur, par l'intermédiaire de 3 touches de commandes et d'un écran à cristaux liquides disposés en face avant,

- avec un système d'exploitation informatique, par l'intermédiaire d'un coupleur fonctionnant par liaison infrarouge bidirectionnelle.

Dans le premier cas, le porteur du badge peut accéder aux informations suivantes, affichées sur l'écran :

- équivalent de dose en cours (depuis une entrée en zone contrôlée),

- débit d'équivalent de dose,

- fichier dosimétrique personnel (accessible sur composition d'un code) constitué des doses mois, trimestre, an et 5 ans).

TABLEAU I

\section{Badge DOSICARD : spécifications}

$\begin{array}{lll}\text { Détection } & \begin{array}{l}\text { Photons X, gamma } \\ \text { et bêta de haute énergie }\end{array} & \text { Dose profonde Hp (10) } \\ \text { Réponse en énergie } & 50 \mathrm{keV}-1,5 \mathrm{MeV} & \text { à mieux que } \pm 20 \% \\ \text { Type de détecteur } & \text { Diode silicium } & \text { compensée en énergie } \\ \text { Etendue de mesure } & \begin{array}{l}\text { Equivalent de dose : } \\ \text { Débit d'équivalent de dose : }\end{array} & \begin{array}{l}1 \mu \mathrm{SV} \text { à } 1 \mathrm{~Sv} \\ 1 \mu \mathrm{Sv} / \mathrm{h} \text { à } 1 \mathrm{~Sv} / \mathrm{h}\end{array} \\ \text { Mémoire } & \text { EEPROM (non volatile) } & \text { sauvegarde : } 10 \text { ans } \\ \text { Communication } & \text { Interface optique } & \text { infra-rouge } \\ \text { Alimentation } & \text { Pile bouton au lithium } & \text { Réf } 2430 \text { (3 volts) }\end{array}$

\subsection{Système d'exploitation}

Bien que le badge puisse être utilisé seul, sans autre support, pour des applications individuelles, ce dernier a été conçu pour être intégré à un système d'exploitation collective des données. Ce système d'exploitation est composé de modules de base organisés autour de micro-ordinateurs de type PC compatible et connectés en réseau. II est pratiquement constitué de deux sous-systèmes : un sous-système d'attribution de badges $(\mathrm{SAB})$, un sous-système d'exploitation dosimétrique (SED). 
a) Sous-système d'attribution de badges (SAB) : il est constitué des éléments suivants :

- un ensemble de badges ;

- un coupleur qui, connecté à un micro-ordinateur de type PC compatible, permet d'effectuer des échanges (lecture/écriture) entre le badge et le micro-ordinateur ;

- un logiciel spécifique fourni sous forme de disquette souple.

Le sous-système $S A B$ réalise les fonctions suivantes:

- initialisation des badges (entrée des données d'identification et de dosimétrie) ;

- modification des données en cours d'exploitation (ex : actualisation des doses légales, changement d'identification de l'agent) ;

- tenue d'un fichier, stocké en mémoire (disque dur), regroupant l'ensemble des badges et des données associées.

b) Sous-système d'exploitation dosimétrique (SED) : lorsque le site de travail est organisé en zones contrôlées, le système proposé comprend, en outre, un sous-système d'exploitation dosimétrique (SED) qui permet la gestion en temps réel des personnels travaillant sur ces sites. Chaque module de base correspondant, par exemple, à un bâtiment, est constitué de coupleurs entrée/sortie de zone gérés localement par un micro-ordinateur de type PC.

Les fonctions réalisées par le SED sont fondamentalement les suivantes :

- gestion en temps réel des données dosimétriques des personnes entrant ou sortant de zone en regard des critères de seuil ;

- gestion de zone comprenant en particulier les modes suivants :

- liste des personnes présentes en zones,

- tri de fichiers suivant des critères tels que noms des agents, noms de sociétés, catégorie des agents, niveaux dosimétriques,

- relevés statistiques d'activité de la zone sur une période de temps donnée à partir d'informations stockées en base de données.

\section{Etat de développement}

Le système DOSICARD a été développé par NOMATEK dans le cadre d'un programme soutenu par le CEA, COGEMA et I'EDF (Département $\mathrm{DSRE}^{1}$ ). Le badge a été conçu en collaboration technique avec I'IPSN ${ }^{2}$ et a passé avec succès les tests d'homologation effectués par le CTHIR ${ }^{3}$. II sera commercialisé dès avril 1993.

Pour tous renseignements complémentaires s'adresser à : NOMATEK, 12, avenue des Prés, 78180 Montigny-le-Bretonneux, Tél. : 306092 46, Fax 30640425.

1 Département Sécurité, radioprotection et environnement.

2 Institut de protection et de sûreté nucléaire.

3 Comité technique d'homologation de l'instrumentation de radioprotection.

NB : Dans un prochain numéro de Radioprotection paraîtra un article sur un produit nouveau similaire proposé par Merlin-Gérin Provence. 